\title{
Una mirada a la internacionalización universitaria desde la perspectiva de la responsabilidad social: discursos de los jóvenes investigadores
}

\author{
A look to the university internationalization from the perspective of social \\ responsibility: young researchers discourses \\ Um olhar sobre a internacionalização universitária na perspectiva da responsabilida- \\ de social: os discursos dos jovens investigadores
}

\author{
Ricardo Gaete Quezada ${ }^{1}$, Miguel Bratos Martín ${ }^{2}$ \\ ${ }^{1}$ Universidad de Antofagasta, rgaete@uantof.cl \\ ${ }^{2}$ Universidad de Valladolid Humanidades
}

\begin{abstract}
RESUMEN
El artículo analiza algunas de las relaciones existentes entre los procesos de internacionalización de las universidades y la responsabilidad social que le cabe en esta materia a dichas instituciones. En la primera parte, se describe conceptualmente la relación entre los procesos de internacionalización universitaria y la cooperación interuniversitaria para el desarrollo, mientras que en la segunda parte se presentan los principales resultados de las entrevistas en profundidad realizadas a un grupo de estudiantes de postgrado en el contexto de su formación como jóvenes investigadores. Los discursos obtenidos reconocen la importancia que posee para los jóvenes investigadores el acceso a becas y ayudas, así como a redes de investigación, en una clara expresión del impacto de los procesos de internacionalización de las universidades. Asimismo, destacan la trascendencia de un buen servicio de Relaciones Internacionales que favorezca la cooperación interuniversitaria.
\end{abstract}

Palabras clave: : cooperación universitaria para el desarrollo, responsabilidad social universitaria, cooperación interuniversitaria, internacionalización de las universidades.

\begin{abstract}
The article discusses some of the relationships between the universities internationalization processes and the social responsibility each institution has in this area. In the first part there is conceptual description of the relationship between the universities internationalization processes and the inter-university cooperation for development. In the second part, the main results of the in-depth interviews made to a group of graduate students in the context of their training as young researchers are presented. The accounts obtained recognize the importance, for young researchers, of having access to scholarships and help, as well as having access to research networks in relation to the impact of the universities internationalization. Furthermore, the transcendence of a good service in the International Relation Department which encourages university cooperation is also mentioned.
\end{abstract}

Key words: university development cooperation, university social responsibility, inter-university cooperation, universities internationalization.

\section{RESUMO}

Analisam-se algumas das relações entre os processos de internacionalização das universidades e da responsabilidade social a elas conferida. Descreve conceitualmente a relação existente entre os processos de internacionalização das universidades e a cooperação interuniversitária para o desenvolvimento. Apresentam-se os principais resultados de entrevistas realizadas com um grupo de estudantes de Pós-graduação, no contexto da formação de jovens investigadores. Por meio dos discursos produzidos, reconhece-se a importância, para jovens investigadores, do acesso à bolsas e auxílios, bem como às redes de pesquisa, como expressão clara do impacto dos processos de internacionalização das universidades. Também se enfatiza a importância do bom funcionamento das Relações Internacionais para favorecer a cooperação entre as universidades.

Palavras chave: cooperação para o desenvolvimento, responsabilidade social da universidade, cooperação interuniversitária, internacionalização das universidades. 


\section{INTRODUCCIÓN}

Las universidades y sus casi nueve siglos de historia en el mundo occidental han debido adaptarse rápidamente a una importante cantidad de cambios sociales, que se expresan en el ámbito universitario en una serie de situaciones entre las que se encuentran la masificación de la educación superior, la transferencia de conocimiento a la industria, la utilización de la nuevas tecnologías de la información y comunicación (NTIC) o la globalización e internacionalización de la actividad universitaria.

En este último ámbito, surge una importante cantidad de indefiniciones o falta de delimitación de una serie de conceptos relacionados con las actividades que las universidades realizan en el ámbito o dimensión internacional (Unceta, 2007; Sebastián, 2002 \& 2009), entre las que se encuentran la internacionalización de las universidades, la cooperación interuniversitaria y la cooperación para el desarrollo. Asimismo Unceta (2007b) reconoce un cambio en las condiciones en las que se debe desarrollar la cooperación al desarrollo, especialmente respecto de las relaciones internacionales y la concepción del desarrollo.

A su vez, Sebastián (2000) señala que la dimensión internacional de las universidades se encuentra integrada por dos grandes componentes que poseen vida propia: la internacionalización y la cooperación internacional. En el caso de la primera, se busca extender el campo de acción de influencia de la universidad mediante una serie de políticas, procesos y actividades para obtener beneficios para la propia institución universitaria que las desarrolla.

En cambio, la cooperación internacional de las universidades, de acuerdo con el propio Sebastián (2000), también posee un carácter instrumental respecto de los objetivos institucionales, y de igual forma como un medio para favorecer su internacionalización. A su vez, posee dos perspectivas de importancia: (1) las universidades como objetivo de la cooperación internacional, orientándose al refuerzo institucional y el beneficio mutuo relacionada con actores y actividades universitarias; y (2) las universidades como agente de la cooperación, ejecutando proyectos de cooperación al desarrollo donde los beneficiarios finales no son las universidades.

De esta manera, resulta interesante observar, tanto desde la perspectiva teórica como empírica, las principales diferencias que es posible establecer entre los conceptos relacionados con la dimensión internacional de las universidades, contextualizadas para el caso específico de este trabajo en el marco de la responsabilidad social universitaria, como el espacio en el cual se desarrollan e insertan estas actividades en la estrategia institucional de cada universidad.

Así, en la primera parte del trabajo, se analizan brevemente algunas de las principales aportaciones conceptuales en el contexto español para la cooperación universitaria al desarrollo y las nociones relacionadas con el mismo.

En la segunda parte del trabajo se presentan los principales resultados del estudio de caso realizado mediante entrevistas en profundidad para una muestra de estudiantes de postgrado iberoamericanos de la Universidad de Valladolid, para identificar los principales discursos relacionados con la cooperación universitaria al desarrollo y la internacionalización de las universidades. 


\section{LA INTERNACIONALIZACIÓN DE LAS UNIVERSIDADES}

Los procesos de internacionalización de las universidades son una expresión creciente dentro de las preocupaciones de estas instituciones en su época moderna, aun cuando desde sus orígenes en la Edad Media las organizaciones universitarias acostumbraban moverse continuamente de un territorio a otro, lo que llevó a los especialistas a acuñar el concepto de universidad de migración para destacar el carácter itinerante o nómade que poseía en sus comienzos (Tamayo, 1987; Moles, 2006; Borrero, 2008), aun cuando como señalan Fernández \& Ruzo (2004) la inexistencia de la figura de países-Estado hace debatible situar en la Edad Media el origen de la internacionalización de las universidades.

Sin embargo, el significado que tiene el concepto de internacionalización en el ámbito de la educación superior no responde a un criterio único, ni una acepción totalmente compartida (Knight, 2005), existiendo confusiones con los conceptos de globalización (Fernández \& Ruzo, 2004; Knight, 2005), con el concepto de interculturalidad (Knight, 2005; Hellstén, 2010), o confundiéndolo con los procesos de transnacionalización de servicios educativos (Didou, 2007), existiendo una importante evolución de la dimensión internacional de las universidades en las últimas décadas (Knight, 2005; Didou, 2007; Hellstén, 2010).

De acuerdo con Knight (2005:13), la internacionalización del quehacer universitario puede ser entendida como "el proceso de integración de la dimensión global, intercultural e internacional en el propósito, las funciones o entrega de la educación superior", precisando con ello que se trata de una ampliación del ámbito local o nacional al cual habitualmente se orientan las universidades, planteando la necesidad de aplicar este proceso de internacionalización a la universidad en su conjunto, no sólo en algunas áreas, ámbitos o fases de su quehacer.

Evidentemente, las motivaciones de las universidades para internacionalizar su quehacer en la actualidad son diferentes, lo que de acuerdo con Fernández \& Ruzo (2004) se puede resumir en cuatro grandes motivos: económico-financiero, culturales, políticos y académicos.

En el caso europeo, por ejemplo, los procesos de internacionalización de la educación superior relacionados con el Espacio Europeo de Educación Superior (EEES) tienen un origen o motivación eminentemente política y económica (Moles, 2006; Sanz, 2006; Mottis, 2008; Hernández, 2010). Para el caso de América Latina, el impulso de la internacionalización de la educación superior responde a los procesos de globalización generados a partir de los acuerdos de integración regional (Didou, 2007). No obstante, a nuestro juicio, dichas motivaciones pueden mostrar dos grandes tendencias: (1) estrictamente económica, que busca proveer a las universidades de nuevos espacios geográficos o nichos para ofrecer sus servicios y capacidades ${ }^{1}$, en un símil con la empresa multinacional (Didou, 2007); y (2) más amplia de carácter social, que intenta ubicar a la universidad en un rol más responsable y comprometida con el desarrollo humano y sustentable tanto de su entorno geográfico más próximo, como respecto de aquellos de países menos desarrollados.

$1 \quad$ Según Hellstén (2010) también es utilizado para atraer estudiantes extranjeros que pagan aranceles y tasas académicas como el caso del sistema de educación superior australiano. 
Las tendencias que hemos mencionado anteriormente no son fácilmente diferenciables si se analiza la internacionalización desde el ámbito educativo, donde según Fernández \& Ruzo (2004) existen tres tipos de internacionalización educativa: movilidad física de los estudiantes al país donde recibirán la educación; movilidad ficticia donde los estudiantes obtienen la titulación en su propio país, la cual es reconocida por una universidad extranjera en virtud a los convenios bilaterales existentes; y, finalmente, la movilidad virtual o educación a distancia.

Existen otros puntos de vista para analizar la dimensión internacional de las universidades como la propuesta por Sebastián (2009), según la cual dicha dimensión posee tres ámbitos de actuación claramente diferenciados, pero entrelazados mutuamente: la cooperación universitaria sensu strictu (interuniversitaria), la cooperación universitaria para el desarrollo y la ampliación o proyección internacional de la oferta académica y de investigación de las universidades.

En lo que se refiere específicamente a la proyección internacional de la universidad, Sebastián (2009:7) señala que "se trata de la ampliación y/o exportación de la oferta y capacidades docentes y de investigación de las universidades [...] corresponde a estrategias de internacionalización focalizadas en la captación de estudiantes, en la exportación de programas docentes y la comercialización en el exterior de las capacidades de investigación y de servicios".

Asimismo, las estrategias de internacionalización de las universidades se estructuran sobre la base de la existencia de acuerdos intergubernamentales de cooperación académica entre países (Didou, 2007; Unceta, 2007), o bien mediante la existencia de acuerdos bilaterales o multilaterales entre universidades, a través de los cuales se establecen los aspectos relacionados con la docencia, investigación y colaboración institucional que materializarán dichos acuerdos, en donde pueden estar contenidos los programas y proyectos de cooperación al desarrollo.

En virtud de los antecedentes expuestos, podemos afirmar que la internacionalización de las universidades es un proceso creciente en las últimas décadas, generados con una base fuertemente exógena basada fundamentalmente en los efectos de la globalización en las universidades.

Sin embargo, las orientaciones que adquieren estos procesos de internacionalización pueden no considerar acciones de cooperación al desarrollo ni de cooperación interuniversitaria, limitándose a ofrecer y proveer servicios académicos en otras latitudes de manera presencial o virtual como su principal y único propósito, más bien como una expresión de la mercantilización de la educación superior que respecto de lo que concierne a la función social de las universidades.

\section{LA COOPERACIÓN UNIVERSITARIA AL DESARROLLO}

En la última década, la cooperación al desarrollo ha dejado de ser exclusividad de los Gobiernos y las Organizaciones No Gubernamentales para el Desarrollo (ONGD) como los principales actores de dichos procesos (Revert, 2007; Unceta, 2007), observándose una creciente pluralidad de instituciones y agentes sociales van sumándose a la ejecución de acciones en estos ámbitos, y en donde las universidades han aumentado 
de manera importante su actividad en este campo ${ }^{2}$, a pesar de lo cual su participación se considera aún como algo novedoso y en proceso de diferenciación respecto del resto de acciones de cooperación internacional de las organizaciones universitarias (Revert, 2007; Unceta, 2007b).

En este contexto, Unceta (2007) reconoce que uno de los ámbitos importantes en donde las universidades deben cooperar es en el fortalecimiento de los sistemas universitarios ${ }^{3}$ de los países en los cuales desarrollan sus acciones de cooperación interuniversitaria, dado que se considera a la enseñanza superior como una herramienta fundamental para que las sociedades menos desarrolladas logren obtener los conocimientos y capacidades necesarias mejorar sus niveles de desarrollo humano y sostenible.

Asimismo, Dominino Crespo (2007) señala que la cooperación universitaria al desarrollo (CUD) se enmarca en el contexto español de la cooperación descentralizada que realizan las administraciones autonómicas y locales, afirmando que la CUD es diferente de la cooperación interuniversitaria o las estrategias de internacionalización de las universidades.

Uno de los elementos diferenciadores de la CUD como expresión descentralizada radica en los procesos de transferencia de conocimientos, los que de acuerdo con Sebastián (2008) poseen un carácter instrumental para obtener resultados medibles en términos de desarrollo económico, social y humano, por lo que dicha transferencia de conocimientos se caracteriza por: (1) la creación o el fortalecimiento de capacidades endógenas en recursos humanos, institucionales, organizativos o productivas; y (2) la aplicación de los conocimientos y tecnologías para la solución de problemas críticos para el desarrollo.

De acuerdo con Arias \& Molina (2008: 46), la universidad en el desarrollo de su función social debe impulsar la solidaridad internacional, implementando políticas institucionales de cooperación al desarrollo, definiendo a la CUD como "una modalidad de actuación relacionada con el ámbito de la solidaridad internacional, coincidiendo su fin último con los objetivos generales de la políticas de cooperación internacional para el desarrollo".

No obstante lo anterior, como nos recuerda Unceta (2007) algunas de las actividades relacionadas con la CUD no tienen una proyección hacia el ámbito internacional, sino que más bien hacia el interior de las propias universidades que realizan dichas actividades, especialmente en el plano de la sensibilización y concienciación de la propia comunidad universitaria respecto de los temas relaciones con el desarrollo humano sustentable.

A su vez, algunos investigadores (Arias \& Molina, 2008; Sebastián, 2009) recomiendan distinguir entre la cooperación universitaria en sentido estricto, la que se asocia con una colaboración interinstitucional entre universidades, mientras que la CUD debe expresarse en un compromiso de la universidad que la ejecuta con los aspectos más esenciales del desarrollo humano tales como la salud, la cultura, el humanismo, la sostenibilidad, el cuidado del medio ambiente y la igualdad entre otras formas de expresión.

2 Desde el punto de vista empírico, el trabajo de Arias \& Molina (2008) para el caso de las universidades madrileñas es un buen ejemplo de dicho crecimiento.

3 No obstante no es el único ámbito, identificando además la promoción de valores solidarios y universalistas, así como el aprovechamiento de sus recursos técnicos y humanos. 
Sin embargo, la Estrategia de Cooperación Universitaria al Desarrollo aprobada por la Conferencia de Rectores de las Universidades Españolas (CRUE), señala que las funciones que las universidades pueden desarrollar en esta temática pueden concentrarse en cinco ejes temáticos: formación y educación, compartir experiencias compartiendo recursos, incidencia en su entorno social, investigación para el desarrollo y transferencia tecnológica.

Respecto de los planteamientos elaborados por la CRUE, resulta importante destacar lo señalado por Sebastián (2003) relacionado con la formación de investigadores, lo que se ve afectado por algunas desarticulaciones entre las becas y ayudas para la formación, el fortalecimiento de las capacidades para la formación de doctorado y la cooperación científica, desajustes que resulta imprescindible corregir para mejorar el impacto de la CUD realizada.

A su vez, el Comité Español Universitario de Relaciones Internacionales (CEURI) a través del Código de Conducta de las universidades en materia de cooperación al desarrollo, en su art. $9^{\circ}$ define a la CUD como "el conjunto de actividades llevadas a cabo por la comunidad universitaria y orientadas a la transformación social y el fortalecimiento académico en los países más desfavorecidos, en pro de la paz, la equidad, el desarrollo humano y la sostenibilidad medioambiental en el mundo".

Otro aspecto importante en el análisis de la CUD se relaciona con su articulación en el funcionamiento institucional de cada universidad, especialmente en lo que se refiere al diseño de una estrategia, respecto de lo cual Unceta (2007) señala que es recomendable "diferenciar y especificar" los contenidos y campos de la cooperación al desarrollo, respecto de aquellos relacionados con la internacionalización de la universidad, complementado con la existencia de órganos especializados en cada materia dentro de la estructura institucional, lo que permitirá avanzar más rápido y con más éxito en la implementación de cada estrategia. Además, respecto de las condiciones internas más adecuadas para posicionar a la CUD Revert (2007) destaca la importancia de "horizontalizar" los objetivos, acciones y propuestas de Cooperación hacia todas las disciplinas y estructuras organizacionales existentes.

\section{COOPERACIÓN INTERUNIVERSITARIA}

Existe una tendencia a confundir a la CUD con los procesos de colaboración interinstitucional que las universidades pueden realizar entre sí a nivel de pares, procesos que de acuerdo con la literatura es recomendable adscribir al concepto de Cooperación Interuniversitaria.

La cooperación interuniversitaria habitualmente obedece a la existencia de diversas carencias que las universidades poseen, tales como insuficiente masa crítica, ausencia de capacidades o recursos que le permitan desempeñarse de forma más autosuficiente (Sanz, 2001), existiendo voluntad de las partes interesadas por cooperar mutuamente, situación plasmada con la firma de convenios específicos de carácter bilateral o multilateral, constituyendo un aspecto relevante de la estrategia de internacionalización de las universidades (Unceta, 2007). De igual manera, las relaciones que se produzcan entre dos o más universidades bajo el alero de la cooperación interuniversitaria presenta un flujo en ambos sentidos, tanto para la universidad que recibe la ayuda como en aquella que proporciona dicho apoyo, lo que deja en evidencia la importancia del rol activo 
de las instituciones involucradas (Sanz, 2001; Sebastián, 2002), situación reflejada en indicadores tales como la obtención de becarios, proyectos de investigación, estudiantes postgraduados, entre otros aspectos.

La bi-direccionalidad de la cooperación interuniversitaria debe fundarse en la confianza mutua y la solidaridad (Arias \& Molina, 2008), especialmente para evitar la "fuga de cerebros" " como uno de los principales problemas de las políticas de internacionalización de las universidades, proponiéndose entre otras medidas la transferencia rápida de conocimientos y tecnologías y ofrecer incentivos para que estudiantes, docentes e investigadores se mantengan en sus universidades de origen.

A su vez, la cooperación interuniversitaria puede desarrollarse en cualquiera de los ámbitos del quehacer universitario (Caparrós, 2001; Sanz, 2001; Unceta, 2001; Sebastián, 2002; Revert, 2007):

- Formación de alumnos (pre y postgrado o doctorado).

- Investigación.

- Difusión cultural y ética hacia la sociedad (foros de debate, publicaciones conjuntas, etc.)

- Gestión universitaria.

- Extensión y vinculación institucional.

- Cooperación internacional para el desarrollo.

Un concepto importante derivado de la cooperación interuniversitaria es la cooperación científica internacional, que según Sebastián (2001) se refiere a la asociación de las universidades para obtener resultados y beneficios mutuos en el ámbito de la investigación y el desarrollo científico, cumpliendo tres grandes funciones: (1) fortalecer las capacidades para la investigación; (2) internacionalizar a los actores que colaboran (personas o instituciones); y (3) contribuir a la cooperación para el desarrollo mediante creación de capacidades para la I+D y el desarrollo de investigaciones conjuntas.

Un elemento central de la cooperación interuniversitaria es la generación o existencia de redes entre las universidades (Bricall, 2000), situación que demanda un cambio cultural en la forma autónoma y autosuficiente en la cual funcionan las universidades actualmente (López Segrera, 2007; Revert, 2007), cuestión que más bien es fácil plantearlo pero completamente difícil de incorporar en el corto y mediano plazo en el "ADN" de las universidades, no obstante ser un aspecto trascendental tanto en el éxito de su internacionalización como respecto de su participación en acciones de cooperación.

\section{LA RESPONSABILIDAD SOCIAL UNIVERSITARIA EN MATERIA DE COOPERACIÓN AL DESARROLLO E INTERNACIONALIZACIÓN}

Las actividades que las universidades desarrollan en el marco de sus procesos de internacionalización se enmarcan dentro del cumplimiento de la función social que tiene

\footnotetext{
4 Un análisis interesante respecto de este tema es realizado por Didou \& Gérard (2009) para el caso Latinoamericano, disponible en: http://www.iesalc.unesco.org.ve/index.php?option=com_fabrik\&c=fo rm\&view=details $\&$ Itemid $=469 \&$ fabrik=10\&rowid=40\&tableid=10\&lang $=e s$
} 
encomendada (Unceta, 2007), razón por la cual existen una serie de campos de actuación que permiten concretar la proyección internacional de las universidades: programas de becas y de movilidad de alumnos y profesores, redes de investigación o de acceso a documentación, cooperación interuniversitaria o el fortalecimiento de los sistemas universitarios en los países en desarrollo, entre otros ámbitos.

En esa perspectiva, Sebastián (2000: 134) señalaba que cuando la universidad participa como agente de la cooperación para el desarrollo debe existir una estrategia institucional que considera esta materia como "un espacio para expresar su responsabilidad social, proyectar y transferir las capacidades y conocimientos de la universidad y obtener diferentes tipos de retornos, incluyendo los financieros", ejecutando proyectos de cooperación para el desarrollo acorde con las capacidades institucionales que se posean.

De esta manera, la universidad en el contexto de la cooperación al desarrollo posee algunas responsabilidades de importancia, tales como otorgar una mayor importancia a la investigación y el debate sobre las condiciones del desarrollo a nivel mundial (Unceta, 2001), reto que debiera ser enfrentado entre otras medidas con la creación de centros o institutos de investigación especializados en la materia.

Estas responsabilidades de las universidades en materia de cooperación al desarrollo señaladas anteriormente, se conectan adecuadamente con los planteamientos que vinculan a la responsabilidad social universitaria (RSU) con la contribución que estas instituciones deben realizar tanto al necesario debate para alcanzar una mejor sociedad, así como respecto de la producción de conocimiento que permita una adecuada transformación social (Chomsky, 2002; Kliksberg, 2009; UNESCO 1998 \& 2009).

También a la universidad le corresponde participar directamente como agente de cooperación, ejecutando proyectos y programas concretos para apoyar las necesidades de las personas de territorios más necesitados, partiendo por establecer procesos de cooperación con otras universidades en países menos desarrollados, que incluyan formación del profesorado, apoyo a la gestión institucional, sistema bibliotecario entre otros aspectos.

Asimismo, la universidad debiera ser capaz de participar en programas y proyectos de cooperación junto a otros actores sociales tales como ONGs, ayuntamientos o empresas, formando consorcios compuestos por una diversidad de instituciones que aúnan esfuerzos, recursos y capacidades para el desarrollo conjunto de dichas iniciativas de cooperación para el desarrollo.

De acuerdo con Arias \& Molina (2008), la cooperación internacional para el desarrollo es uno de los campos que comprende la RSU, por lo que las actividades que las instituciones de educación superior desarrollen en esta temática tienen que transformarse en un aspecto integral de su misión institucional, que complemente su quehacer científico mediante el desarrollo de su función social, evitando que estas instituciones sean consideradas solamente como industrias del conocimiento o fábricas de profesionales.

De esta manera, la universidad como institución tiene una función social vinculada a la solidaridad internacional y la cooperación al desarrollo como parte de sus desafíos para contribuir a la transformación social (Unceta, 2007; Arias \& Molina, 2008), por lo que la internacionalización de la universidad a través de acciones de cooperación para el desarrollo le permitirá cumplir con los retos de su RSU en este ámbito, contribuyendo al establecimiento de relaciones entre personas, instituciones, regiones y países, como un claro aporte también al fortalecimiento del capital social especialmente en aquellas zonas con menor desarrollo. 
Finalmente, Unceta (2007: 45) identifica como uno de los ámbitos que las universidades deben considerar en sus estrategias de cooperación al desarrollo a la promoción de los valores solidarios y universalistas, tanto en su interior, especialmente entre los jóvenes que forma, así como hacia el resto de la sociedad mediante la realización de una serie de acciones concretas: (1) incorporación a sus programas docentes y de investigación de las preocupaciones por la equidad, el desarrollo humano y la sostenibilidad; (2) promoción del voluntariado a través de la generación de espacios de debate y sensibilización; y (3) participación en programas y proyectos de cooperación para el desarrollo.

\section{METODOLOGÍA}

El objetivo de este estudio es analizar los discursos relacionados con los procesos de internacionalización universitaria que formulan una muestra de estudiantes iberoamericanos de postgrado de la Universidad de Valladolid, específicamente respecto de la vinculación de los mismos con aspectos propios de la dimensión internacional de las universidades, o con los elementos distintivos de los procesos de cooperación interuniversitaria y de cooperación para el desarrollo.

El estudio realizado es de carácter cualitativo, concentrándose en la búsqueda del significado que tiene para los estudiantes de postgrado entrevistados la internacionalización universitaria, especialmente respecto de su relación con la responsabilidad social que poseen las instituciones de educación superior en esta materia.

La técnica de recolección de la información utilizada es la entrevista en profundidad, con el propósito de recoger los discursos relacionados con las experiencias, vivencias y perspectivas que los entrevistados poseen respecto de los procesos de internacionalización de la educación superior, así como respecto de las formas en las cuales su experiencia como jóvenes investigadores se ve afectada por dichos procesos.

La muestra corresponde a un total 11 estudiantes iberoamericanos de postgrado de la Universidad de Valladolid en España, en su condición de personal investigador en formación, definida como "aquellos graduados universitarios que sean beneficiarios de programas de ayuda dirigidos al desarrollo de actividades de formación y especialización científica y técnica a través, como mínimo, de los correspondientes estudios oficiales de doctorado", definición establecida por el Real Decreto 63/2006 que aprueba el Estatuto del Personal Investigador en Formación en España.

Para realizar el examen de los discursos obtenidos se utilizará la técnica de análisis de contenidos de tipo comparativo con base gramatical, para lo cual se definen previamente las respectivas unidades de análisis y sus correspondientes categorías en el siguiente cuadro:

\begin{tabular}{|c|c|}
\hline $\begin{array}{c}\text { Unidades deAnálisiso } \\
\text { Registro }\end{array}$ & Categorías deAnálisis \\
\hline \multirow{3}{*}{$\begin{array}{l}\text { Proyección Internacional de } \\
\text { las universidades }\end{array}$} & Programas de B ecas y A yudas para la movilidad. \\
\hline & Redes de Investigación. \\
\hline & O ferta de formación de postgrado. \\
\hline
\end{tabular}




\begin{tabular}{|c|l|}
\hline \multirow{2}{*}{$\begin{array}{c}\text { Cooperación } \\
\text { Interuniversitaria }\end{array}$} & Convenios bilaterales o multilaterales. \\
\cline { 2 - 2 } & Organos administrativos especializados. \\
\hline Cooperación Universitaria al & Fortalecimiento de sistemas universitarios en países en desarrollo. \\
\cline { 2 - 2 } Desarrollo & Transferencia de conocimientos y técnicas. \\
\hline
\end{tabular}

Fuente: Elaboración propia

Cuadro 1. Unidades y Categorías del Análisis de Contenido

Finalmente, la hipótesis del estudio plantea que los jóvenes investigadores entrevistados en la muestra relacionan su experiencia de formación de postgrado con un contexto propio de la proyección internacional de las universidades, más que con elementos relacionados con la cooperación para el desarrollo que deben realizar las instituciones de educación superior en el marco de su responsabilidad social.

\section{RESULTADOS}

En términos generales, los discursos obtenidos en las entrevistas describen de manera mucho más constante aspectos relacionados con la proyección de la dimensión internacional de las universidades, así como respecto de la cooperación interuniversitaria, existiendo una frecuencia más bien baja de discursos relativos a la cooperación universitaria al desarrollo.

\subsection{PROYECCIÓN INTERNACIONAL DE LAS UNIVERSIDADES}

Respecto de esta primera unidad de análisis, el discurso de los jóvenes investigadores es claro y concluyente respecto de la importancia que tiene para la perspectiva de su experiencia los aspectos relacionados con las becas y ayudas, así como la existencia y acceso a redes de investigadores, factores que les proporcionan importantes oportunidades y apoyo para desarrollar su carrera como tales.

\section{a) Becas y ayudas}

En esta primera categoría de análisis, los discursos de los jóvenes investigadores se distribuyen fundamentalmente en el reconocimiento de la importancia que tiene poseer una beca para poder desarrollar su carrera como jóvenes investigadores, y por otra parte, los entrevistados hacen referencia a las formas de acceso a las becas y ayudas para investigar.

En el caso de la importancia de contar con becas y ayudas, se reconoce que muchas veces existen dificultades para acceder a las mismas, situación que puede poner en peligro la formación de un investigador ante la falta de oportunidades de acceso:

“...creo que el principal problema para los investigadores es la manutención de su estancia, son las becas...muchos de ellos no tienen esta ayuda de su gobierno, de su país de origen y también no tienen en el país de destino para desarrollar su trabajo..." (Entrevistado $\mathrm{N}^{\circ} 3$ ) 
“...para continuar investigando tienes que tener principalmente dinero. Si no hay una beca que te respalde, no puedes hacer nada...” (Entrevistado $\mathrm{N}^{\circ} 10$ )

Además, ambos discursos señalan implícitamente una condición importantísima para la formación de un investigador: la dedicación exclusiva. Esta situación se relaciona con la necesidad de evitar la doble condición de investigador en formación y tener que trabajar en otro ámbito o sector para obtener los recursos económicos para poder subsistir, algo que también estará condicionado por la cuantía de la beca y el costo de vida del lugar donde se estudia entre otros factores condicionantes:

“...tener la oportunidad para salir de tu país para estudiar al fin un doctorado y hacer investigación este pues... para mí era un sueño, quería si o si venir para poder este dedicarme solamente a estudiar, a investigar y no hacer dos o tres cosas como lo hacía en mi país: estudiar, trabajar...” (Entrevistado №5)

“...pues depende de si tienes una beca que te lo permita o no. Si no tienes beca, tienes que trabajar, y hay mucha gente en esa situación, en situación de que está haciendo Máster o lo que sea e incluso, aunque tenga becas del Ministerio necesitan trabajar para...sobrevivir, entonces tienen que trabajar y tienen que dedicarse a trabajar y estudiar al mismo tiempo e investigar porque les interesa..." (Entrevistado $\mathrm{N}^{\circ} 11$ )

El otro aspecto interesante en los discursos se relaciona con los mecanismos de acceso a las becas y ayudas utilizados por los jóvenes investigadores, los cuales mayormente a la existencia de un profesor que patrocina y apoya al postulante en este proceso:

“...mi beca es del Banco de Santander, ves como digamos.... los profesores están.... tienen el conocimiento de estas becas y los mismos profesores son las ofrecen a los candidatos son los mismos profesores entonces pues yo creo que.....que si hay un esfuerzo para apoyar..." (Entrevistado $\mathrm{N}^{\circ}$ 9)

La influencia del profesor que patrocina o avala al joven investigador en la búsqueda de obtener una beca es tan importante que puede ser capaz de determinar el lugar seleccionado para realizar el proceso de formación, lo que va dando vida a las redes de investigadores:

“...yo elegí Valladolid este....porque había una persona que podía recomendarme y había un poco de más posibilidades de conseguir la beca acá, si optaba por Madrid, pues no conozco a nadie y no había quién me podía recomendar..." (Entrevistado $\mathrm{N}^{\circ} 5$ )

“...para las becas en general conviene pedirlas con el profesor asociado al Máster en el que estas. Entonces supongo que la gente...o sea toda la gente que da clase allí prácticamente son de Valencia, de Valencia y Castellón pero la parte que yo voy a hacer son de Valencia, y supongo que pediré la beca con alguien de Valencia..." (Entrevistado $\mathrm{N}^{\circ} 11$ )

Finalmente, el proceso de postulación y obtención de una beca para los jóvenes investigadores, especialmente de los latinoamericanos, también se ve enfrentado a la necesidad de superar algunos aspectos administrativos a los que las universidades deben prestar más atención para poder reclutar más y mejores candidatos:

“...al solicitar becas si, por mi titulación es brasileña he tenido algunos problemas por no tenerla homologada, entonces hay algún tipo de becas que necesitan la homologación del título, y ha sido en verdad la única barrera que sentí que para solicitar algunas becas..." (Entrevistado $\mathrm{N}^{\circ} 4$ ) 
De esta manera, los resultados obtenidos en esta primera categoría de análisis nos plantean importantes conexiones con los aspectos teóricos respecto de la internacionalización de las universidades, especialmente en lo que se refiere a la captación de estudiantes, la movilidad física de los estudiantes y la importancia de los programas de becas.

\section{b) Redes de investigación}

En esta segunda categoría de análisis de la internacionalización de las universidades, el impacto que tiene la movilidad académica en la generación de redes de investigación es muy importante en el discurso de los entrevistados, especialmente respecto de los vínculos generados por los profesores españoles en América Latina a través de sus visitas a diferentes países de la Región, lo que posteriormente permite a los jóvenes investigadores conseguir apoyo para iniciar su experiencia internacional:

“...lo conocí allá en Perú y por intermedio de él es que pude venir en una oportunidad acá a Valladolid a un curso, y luego este pedirle apoyo para conseguir algún tipo de ayuda y venirme a investigar..." (Entrevistado $\mathrm{N}^{\circ} 5$ )

“...pero también es cierto que mi situación es especial porque ya hace más de 10 años que estoy en contacto con profesores de Valladolid que han ido a Argentina a Rosario a mi universidad a trabajar, y creo que eso también facilitó las cosas..." (Entrevistado №7)

La generación de redes entre los investigadores consolidados y los jóvenes investigadores, no sólo tiene efectos relacionados con los contactos previos necesarios para comenzar este proceso, sino que, además, se transforma en un "polo de atracción" de otros jóvenes debido al prestigio y experiencia de estos investigadores consolidados:

“...yo no conocía Valladolid, no conocía España, era la primera vez que estaba en Europa, y pues bueno con la trascendencia de los profesores que estaban allí, que son personas que han publicado muchos artículos, que tienen años dedicados a la investigación..." (Entrevistado $\mathrm{N}^{\circ}$ )

“...creo que la diferencia que hay acerca de la UVa [Universidad de Valladolid] tiene como base el área de investigación en sí, entonces si tú tienes investigadores que tienen una especialidad muy enfocada y sabes que ese investigador tiene clases aquí entonces claro esa es la razón, aunque también sabemos que en universidades como Madrid haya también otros investigadores y grupos de investigación muy enfocados..." (Entrevistado No8)

Por último, los discursos de los jóvenes investigadores entrevistados identifican los efectos que tiene para sus posibilidades profesionales no disponer de redes de investigadores que los apoyen:

“...en Perú hay chicos muy buenos con talento pero lamentablemente no tienen este... digamos personas que les puedan recomendar para que opten por una de estas becas, porque primero hay que hacer un maestría y trabajar con alguien y recién allí tener contacto con una persona..." (Entrevistado $\mathrm{N}^{\circ} 5$ )

“...el investigador consolidado cuenta con la ventaja que ya es...y y ha estado investigando, ya tiene sus trabajos, ya tiene gente que le apoya, ya tiene un sustento económico importante, ha sido reconocido en mayor o menor medida, y el joven investigador hasta que pase a ser investigador consolidado...y en un país como España pues a lo mejor no pasa nunca...a no ser que tengas alguien que realmente te aprecie y pelee por ti que va a haber pocos..." (Entrevistado $\left.\mathrm{N}^{\circ} 10\right)$ 
De esta manera, los discursos obtenidos en esta categoría confirman la importancia que tienen las redes de investigación para el reclutamiento de jóvenes investigadores, al igual que para el acceso a becas y ayudas, lo que se transforma en un importante apoyo a la internacionalización de las universidades, y un elemento diferenciador frente al resto de instituciones de educación superior que desarrollan estrategias similares.

\section{c) Oferta de formación de postgrado}

En el caso de esta temática, los jóvenes investigadores reconocen la importancia que tiene una adecuada oferta de formación de postgrado, especialmente para eliminar o disminuir algunas barreras de acceso que pueden existir respecto de determinadas áreas científicas o zonas geográficas:

“...la distinguiría mucho a razón de cómo una persona investiga en mi país, muchos no lo hacen primero por razones económicas y luego porque existe mucho digamos.... muchos requisitos para que una persona pueda hacer un postgrado, incluso hay demasiado este digamos requisitos para ingresar a la universidad, lo que no veo acá en Europa...” (Entrevistado №5)

“...para filosofía política solamente hay máster en Valencia y Barcelona y me gusta Valencia como ciudad, para estar, porque el tema de los máster también está muy disgregado y dependiendo de que quieras hacer solamente tienes máster en determinados sitios en España y entonces es, te tienes que ir básicamente a donde lo den...” (Entrevistado $\mathrm{N}^{\circ} 11$ )

De esta manera, la oferta de postgrados que realice una universidad inserta dentro de su estrategia de internacionalización puede contener una importante dimensión social, que intente responder a las problemáticas de acceso reconocidas en estos discursos y no únicamente diseñar una oferta de este tipo bajo criterios puramente económicos o de mayor visibilidad institucional.

\subsection{COOPERACIÓN INTERUNIVERSITARIA}

Los discursos referidos a aspectos de cooperación interuniversitaria son más bien bajos e indirectos, en parte porque la mayoría de los entrevistados desarrolla su proceso de formación como joven investigador en un plano especialmente individual, desconectado de relaciones institucionales con su lugar de origen.

\section{a) Convenios bilaterales o multilaterales}

Respecto de este primer aspecto, sólo en un caso la existencia de un convenio se transformó en un factor importante para impulsar y apoyar la decisión de realizar su experiencia de postgrado en la UVa:

“...elegí Valladolid por el castellano y porque tiene convenio, no con la universidad que hice mi carrera, sino por la universidad de mi ciudad, porque hice mi carrera en otra ciudad y entonces el convenio aunque de momento no lo necesité y nada de esto, pero siempre es una ayuda para tener relaciones entre mi ciudad, la universidad de mi ciudad y la de aquí..." (Entrevistado No4)

Sin embargo, este discurso es interesante porque visualiza el impacto social de los convenios, más allá de la simple relación entre universidades, extendiéndolo a las posibles vinculaciones que dicho vínculo puede generar para ambas ciudades, un aspecto 
especialmente relevante a tener en cuenta desde la perspectiva de la responsabilidad social de una universidad cuando establece este tipo de acuerdos interuniversitarios.

\section{b) Órganos administrativos especializados}

En este segundo factor, los discursos se dividen claramente en aquellos que reconocen la importancia del apoyo del órgano encargado de las Relaciones Internacionales de la universidad; los discursos que formulan claras demandas a la misión que cumplen estas instancias administrativas respecto de su experiencia como joven investigador, así como los discursos que formulan críticas a su labor.

“...la llegada acá fue bastante buena, de relaciones internacionales me ayudaron, me consiguieron un lugar donde pasar el primer tiempo y la verdad es que han sido un apoyo... bueno todo el tiempo..." (Entrevistado $\mathrm{N}^{\circ} 1$ )

La necesidad de contar con un apoyo institucional para realizar los trámites administrativos y consulares se transforman en las principales demandas de los estudiantes de postgrado hacia el Servicio de Relaciones Internacionales de la universidad:

“...los profesionales que están aquí para ayudarte con el sistema, me parece que ponen muchísimo interés, pero hay siempre dificultad de la comprensión de los que vienen, de cómo desarrollar estas gestiones, como resolver sus problemas, sobre todo cuando tienen la barrera de la lengua, pero siempre hay problemas administrativos...” (Entrevistado $\mathrm{N}^{\circ} 3$ )

“...las autoridades de la universidad y junto con las de Relaciones Internacionales de este país pues deben tener conocimiento para que la Embajada en Perú o en cualquier otro país latinoamericano den facilidades para eso, porque.....es una cosa terrible los papeles de verdad..." (Entrevistado No5)

Cabe recordar que los entrevistados declararon anteriormente solucionar los inconvenientes enfrentados en los procesos de obtención de becas mediante el apoyo individual de profesores de la universidad, situación no replicable a otros problemas administrativos como la obtención de visados, donde se hace imprescindible el concurso de una entidad administrativa que oficialice la documentación necesaria para este tipo de trámites, especialmente cuando existen becas de por medio. En cuanto a las críticas, muy relacionado con lo anterior, la necesidad de que los Servicios de Relaciones Internacionales desarrollen buenos sistemas de difusión de información resulta evidente:

“...entonces para hacer intercambio a otro país, y hay estas becas pero las Relaciones Internacionales no nos informan muy bien acerca de eso, y tampoco acerca de cursos de idiomas para iberoamericanos que no provienen de países de habla hispánica, éstas son las dificultades involucradas dentro de la carencia de información..." (Entrevistado No8)

De esta forma, la importancia de órganos especializados que apoyen los procesos de cooperación interuniversitaria no tiene discusión, otra cuestión diferente es la eficacia y calidad con la que pueden desarrollar su labor.

\subsection{COOPERACIÓN UNIVERSITARIA PARA EL DESARROLLO}

En esta tercera unidad de análisis es donde se registran la menor cantidad de discursos asociados, probablemente por la fuerte connotación individual de la experiencia 
como joven investigador señalada anteriormente, y además debido a la falta de redes de investigación y convenios gubernamentales que les permitan reinsertarse en los sistemas universitarios y de I+D de sus países o lugares de origen.

\section{a) Fortalecimiento de sistemas universitarios en países en desarrollo}

Como hemos señalado en la discusión teórica, las universidades que desarrollan acciones de CUD en sus estrategias de internacionalización deben orientarse a fortalecer el sistema universitario de los países en desarrollo, algo que, sin embargo, no puede concretarse sin el compromiso de las personas y sus reales intereses individuales por contribuir con este objetivo:

“...para mí significa ser un joven investigador pues una persona que no solo se va a dedicar a la docencia sino que a velar por los intereses del país ¿no? Empezando desde la universidad ¿no? Ayudar al desarrollo como universidad y luego aportar para el desarrollo del país...” (Entrevistado N 5 )

El impacto y la contribución que pueda realizar un joven investigador al momento de reinsertarse en su lugar de origen, estará fuertemente condicionado por el "efecto mateo"5 respecto a la importancia del reconocimiento adquirido por los científicos:

“...pues para mí es muy importante hacerlo [sic] la investigación fuera del Brasil, en Europa porque el peso en Brasil es más significativo de los jóvenes investigadores que hay allí, todavía se valora mucho tener la experiencia afuera..." (Entrevistado $\mathrm{N}^{\circ} 4$ )

“...pues sí, sí que es importante tener este un grado de doctor, al menos en mi país sí, porque si llegas a hacer una maestría igual no te sirve de mucho, te sirve si para conseguir un trabajo de docencia, y alli te quedas, no progresas..." (Entrevistado $\mathrm{N}^{\circ} 5$ )

De esta manera, resulta importante generar estrategias de internacionalización que ofrezcan oportunidades a los jóvenes talentos para perfeccionarse en el extranjero, siendo la temática de la movilidad estudiantil y académica un aspecto relevante del impacto social de estas acciones cuando se hable de la proyección internacional de las universidades.

\section{b) Transferencia de conocimientos y técnicas}

En este segundo aspecto que debiera formar parte de las acciones de CUD, los discursos obtenidos reconocen dicha importancia, tanto para el país donante como para el receptor:

“...un espacio de intercambio de experiencias, de saberes, de conocimientos entre diversos países, por algo estoy acá también, me interesa digamos...conocer cuál es el estado de la cuestión aquí en España...” (Entrevistado $\mathrm{N}^{\circ} 1$ )

“...entonces involucrar toda esta gente aquí en las actividades de investigación creo que es una dimensión para la España sobre todo, importantísimo porque son países que tienen algo de su cultura, su formación entonces tienen de alguna forma algún tipo de ganancia, entonces esto creo que enriquece sobremanera la investigación..." (Entrevistado №3)

5 Este efecto es descrito por Robert K. Merton (1977) respecto al mayor reconocimiento que reciben los investigadores consolidados, en desmedro de los investigadores más jóvenes o menos prestigiados, quienes habitualmente sufren una serie de postergaciones debido a la falta de reconocimiento. 
Asimismo, las posibilidades de acceder al dominio de técnicas y conocimientos que no se tienen al alcance habitualmente en sus lugares de origen:

“...estando aquí me parece un máster...enfocado a lo que yo quería, y me ha ampliado los conocimientos porque la verdad no tenía ninguna de las herramientas...” (Entrevistado №2)

De esta manera, debiera hablarse no sólo de una transferencia sino que también de un intercambio tanto de conocimientos y técnicas, así como de experiencias y vivencias humanas y culturales como parte de los efectos de las CUD.

\section{CONCLUSIONES}

En virtud de los antecedentes teóricos analizados, las estrategias de internacionalización universitaria compuestas por la oferta de servicios docentes y de investigación en otras latitudes geográficas, pueden considerar también acciones enmarcadas en los conceptos de cooperación interuniversitaria y cooperación para el desarrollo, decisión que estará fuertemente influenciada por una adecuada asunción de la responsabilidad social que le cabe a cada universidad en dichos ámbitos.

También desde la perspectiva teórica, resulta importante lograr diferenciar y equilibrar los objetivos y acciones de la internacionalización universitaria, la cooperación interuniversitaria y la CUD, situación que incluso desde una perspectiva conceptual no siempre resulta tan sencillo y evidente de alcanzar.

Los aspectos mencionados en los párrafos anteriores tienen directa relación con las motivaciones que las universidades poseen para emprender procesos de internacionalización, donde muchas veces puede primar lo económico y el desarrollo institucional por sobre el compromiso socialmente responsable de las universidades.

Desde la perspectiva empírica, los discursos obtenidos claramente reconocen la importancia de las becas y ayudas, las redes de investigación o la existencia de órganos administrativos especializados, en una clara referencia a la proyección internacional de las universidades y la cooperación interuniversitaria, existiendo menos referencias discursivas acerca de la CUD.

Más en detalle, se constata en los discursos obtenidos una clara conexión entre el acceso a las becas y ayudas con la existencia de redes de investigación que posibilitan a los jóvenes investigadores la información, apoyo y acceso a la obtención de estos beneficios, siendo clave para las universidades que desean internacionalizarse el rol que juegan los investigadores consolidados en el reclutamiento de los jóvenes talentos que desean iniciar una carrera como investigadores.

Sin embargo, también existen discursos que reconocen que muchos jóvenes investigadores no tienen acceso a estas redes para obtener una beca, lo que muchas veces incide en que este tipo de experiencias se desarrollen desde una perspectiva más bien individual o privada por parte de los estudiantes de postgrado, situación que afecta notablemente las posibilidades de éxito de las estrategias de cooperación interuniversitaria y especialmente de CUD, como evidencia la frecuencia de discursos obtenidos en este estudio de caso.

Finalmente, en base a los discursos obtenidos en las entrevistas es posible identificar algunas tareas o ámbitos en los cuales resulta necesaria la expresión de una mayor RSU en materia de internacionalización: aplicación de una mirada triple hélice a los procesos 
de suscripción convenios en el ámbito internacional; apoyo a los estudiantes de postgrado reclutados en los trámites administrativos y legales asociados a esta experiencia de movilidad estudiantil; y priorizar en el diseño de la oferta de postgrados hacia zonas geográficas y áreas del conocimiento con mayores problemas de acceso a este tipo de posibilidades.

\section{REFERENCIAS BIBLIOGRÁFICAS}

Arias, S. \& Molina, E. (2008). Universidad y cooperación al desarrollo. La experiencia de las universidades de la ciudad de Madrid. Madrid: Catarata.

Borrero, A. (2008). La Universidad. Estudios sobre sus orígenes, dinámicas y tendencias. Vol.1. Bogotá: Pontificia Universidad Javeriana.

Bricall, J. (2000). Universidad 2000. Madrid: CRUE.

Caparrós, A. (2001). Reflexiones sobre los entornos universitarios para los intercambios y la movilidad. En Sebastián, J. (coord.) La universidad como espacio para la cooperación iberoamericana (pp. 71-73). Valladolid: Secretariado de Publicaciones Universidad de Valladolid.

Comité Español Universitario de Relaciones Internacionales (CEURI). (2006). Código de Conducta de las universidades en materia de cooperación al desarrollo. [Acceso: 17 de junio 2010]. http://campus.usal.es/ cooperacion/normativa/crueCodigoConducta.pdf

Conferencia de Rectores de las Universidades Españolas (CRUE). (2000). Estrategia de Cooperación Universitaria al Desarrollo. [Acceso: 22 de julio 2010] http://www.ucm.es/cont/descargas/ documento2997.pdf

Chomsky, N. (2002). Los límites de la globalización. Barcelona: Ariel.

Didou, S. (2007). Internacionalización de la educación superior y provisión transnacional de servicios educativos en América Latina: del voluntarismo a las elecciones estratégicas. En IESALC-UNESCO Informe sobre la Educación Superior en América Latina y el Caribe 20002005. La metamorfosis de la educación superior (pp. 21-32). [Acceso: 26 de julio de 2010] http://www.oei.es/salactsi/informe_educacion_superiorAL2007.pdf

Dominino Crespo, D. (2007) Perfiles de la universidad española en cooperación para el desarrollo. Una aproximación a las relaciones con las ONGD. España: Centro de investigación y cooperación para el desarrollo CIDEAL [Acceso: 17 de junio 2010] http://www.cideal.org/ news_pdf/cooperacion_universitaria_OK.pdf

Fernández, S. y Ruzo, E. (2004) Los procesos de internacionalización y globalización en la Educación Superior: un análisis de los países de la OCDE. Revista de Educación, n. 335, 385-413. [Acceso: 25 de julio de 2010] http://www.revistaeducacion.mec.es/re335/re335_25.pdf

Hellstén, M. (2010) Internacionalizar la universidad. Cuadernos de Pedagogía, n. 403, 32-35.

Hernández, F. (2010) La declaración de Bolonia, ¿oportunidad o problema? Cuadernos de Pedagogía, n. $403,24-27$

Kliksberg, B. (2009) Los desafíos éticos en un mundo paradojal: el rol de la universidad. Revista del CLAD Reforma y Democracia, n. 43. [Acceso: 02 de abril de 2010] http://www.clad.org/portal/ publicaciones-del-clad/revista-clad-reforma-democracia/articulos/043-febrero-2009-1/kliksberg

Knight, J. (2005) An internationalization model: responding to new realities and challenges. En De Wit, Hans; Jaramillo, Isabel; Gacel-Ávila, Jocelyne \& Knight, Jane (eds.), Higher Education in Latin America. The international dimension (pp. 1-38). The World Bank. [Acceso: 25 de Julio de 2010] http://siteresources.worldbank.org/EXTLACREGTOPEDUCATION/Resources/ Higher_Ed_in_LAC_Intnal_Dimension.pdf

López Segrera, F. (2007) Educación Superior Internacional Comparada, Global University Network for Innovation (GUNI). [Acceso: 25 de julio de 2010] http://mt.educarchile.cl/mt/jjbrunner/ archives/Lopez.pdf 
Merton, R. (1977). La sociología de la ciencia, Tomos I y II, Madrid: Alianza.

Moles, R. (2006). ¿Universidad S.A.? Público y privado en la educación superior, Barcelona: Ariel.

Mottis, N. (2008). Bologna and business education: far from a model, just a process for a while. En Mazza, C., Quattrone, P. \& Riccaboni, A. (Eds.), European universities in transition. Issues, models and cases (pp. 93-112). Cheltenham: Edward Elgar.

Revert, X. (2007) .La Cooperación Técnica Universitaria: Rasgos del ámbito valenciano. Àgora Revista de Ciencias Sociales, n. 16, 139-153.

Sanz, F. (2006). La mercantilización de la educación como escenario mundial del Espacio Europeo de Educación Superior. Revista Educación XX1, n. 9, 57-76.

Sanz, J. (2001). La cooperación en los marcos interuniversitarios. En Sebastián, Jesús (coord.), La universidad como espacio para la cooperación iberoamericana (pp. 57-60). Valladolid: Secretariado de Publicaciones Universidad de Valladolid.

Sebastián, J. (2009). Oportunidades y desafíos de la Cooperación Universitaria al Desarrollo. En Ingeniería sin Fronteras Proyectos fin de Carrera en Cooperación para el Desarrollo (pp. 5-16). [Acceso: 22 de julio de 2010] http://admin.isf.es/UserFiles/File/apd/publicaciones/ publicacion_PFC.pdf

Sebastián, J. (2008) La transferencia de conocimientos en la cooperación al desarrollo. Revista Arbor, n. 732, 719-728.

Sebastián, J. (2003). Estrategias de cooperación universitaria para la formación de investigadores en Iberoamérica. Madrid: OEI.

Sebastián, J. (2002). Oportunidades e iniciativas para la cooperación iberoamericana en educación superior. Revista Iberoamericana de Educación, n. 28, 197-229.

Sebastián, J. (2001). Análisis y oportunidades de la cooperación científica entre España y América Latina. En Sebastián, Jesús (coord.). La universidad como espacio para la cooperación iberoamericana (pp. 103-121), Valladolid: Secretariado de Publicaciones Universidad de Valladolid.

Sebastián, J. (2000) Modalidades y tendencias de la cooperación internacional de las universidades. Revista Española de Desarrollo y Cooperación, n. 5, 125-144.

Tamayo, R. (1987). La Universidad, epopeya medieval. México D.F.: UNAM.

Unceta, K. (2007). La cooperación al desarrollo en las universidades españolas. Madrid: AECI Ministerio de Asuntos Exteriores y de Cooperación.

Unceta, K. (2007b). Cooperación al desarrollo y asistencia técnica: el papel de la universidad. Àgora - Revista de Ciencias Sociales, n. 16, 39-52.

Unceta, K. (2001). Universidad como ámbito para la solidaridad y la cooperación al desarrollo. En Sebastián, Jesús (coord.). La universidad como espacio para la cooperación iberoamericana (pp. 123-128). Valladolid: Secretariado de Publicaciones Universidad de Valladolid.

UNESCO (2009). La nueva dinámica de la Educación Superior y la investigación para el cambio social y el desarrollo. Conferencia Mundial sobre la Educación Superior, Comunicado de Prensa. [Acceso: 18 de mayo de 2010] http://www.unesco.org/education/WCHE2009/comunicado_es.pdf

UNESCO (1998). Autonomía, responsabilidad social y libertad académica. VII Conferencia Mundial de Educación, Paris Francia. [Acceso: 25 de julio 2009] http://unesdoc.unesco.org/ images/0011/001135/113549so.pdf 